\title{
Preparation of Biology Review and Virtual Experiment/Training Videos to Enhance Learning in Biochemical Engineering Courses
}

\section{Dr. Jacob James Elmer, Villanova University}

Dr. Elmer earned dual B.S. degrees in Biology and Chemical Engineering from the University of Missouri Rolla in 2003 and obtained a PhD in Chemical Engineering from Ohio State University in 2007. After a short posdoc at Arizona State University and some adjunct teaching at Grand Canyon University, he secured an Assistant Professorship at Villanova University in the Chemical Engineering department. He currently teaches heat transfer and several biochemical engineering electives (Lab Techniques, Protein Engineering, etc.). His research focuses on developing novel blood substitutes and optimizing gene therapy treatments.

\section{Dr. Noelle K Comolli, Villanova University}

Zuyi (Jacky) Huang, Villanova University

Zuyi (Jacky) Huang is an Assistant Professor in the Department of Chemical Engineering at Villanova University. He teaches Chemical Process Control (for senior students) and Systems Biology (for graduate students) at Villanova. He is enthusiastic in applying innovative teaching methods in class to educate students with modeling and control skills. His research is focused on developing advanced modeling and systems analysis techniques to manipulate microbial biological systems for generating biofuels from wastewater and for combating biofilm-associated pathogens. His BESEL group developed the first model for microbial desalination cells and the first metabolic modeling approach for quantifying the biofilm formation of pathogens. 


\title{
Preparation of Biology Review and Experiment/Training Videos to Enhance Learning in Biochemical Engineering Courses
}

\begin{abstract}
$\underline{\text { Abstract }}$
Two major challenges are inherent in any biology-related engineering subject (e.g. biochemical engineering, biomechanical engineering, or civil/environmental engineering) - (1) the student must have a good understanding of fundamental biology concepts to fully comprehend the advanced engineering concepts and (2) most of the techniques discussed in these courses are too time-consuming, hazardous, or expensive to demonstrate in a classroom setting. We have addressed these issues by preparing a series of videos that review basic biology concepts and demonstrate several common biochemical engineering (BioChE) techniques and experiments. The primary goal of the review videos is to ensure that students have a firm grasp on basic biology concepts to help them fully understand the more advanced BioChE topics discussed in class. These videos can also be assigned before class, allowing us to spend more class time discussing new and advanced BioChE concepts. The goal of the experiment videos is to help us expose the students to state-of-the-art experimental techniques in lecture-based classes or to help them better prepare for the experiments that they conduct in lab-based courses. We have also used these videos to streamline the training process for undergraduate and graduate students performing research in our labs. Some of the review and experiment videos have already been utilized in our BioChE courses and we have evaluated their effectiveness with student surveys. The feedback from these surveys is highly positive and indicates that the videos help to enhance student learning.
\end{abstract}

\section{$\underline{\text { Introduction }}$}

Since the advent of broadband internet provided on-demand video capability to students in the late 1990's, instructors have been experimenting with the use of online lessons and videos to enhance student learning both inside and outside of the classroom (e.g. flipped classrooms ${ }^{1}$ and distance learning ${ }^{2}$ ). Online videos and animations have been shown to be especially effective for teaching molecular biology concepts ${ }^{3,4}$, which are often too small to see with the naked eye and difficult to conceptualize. ${ }^{5}$ Several organizations have already created full length online videos for biology topics that are available free of charge, including the BioVisions series from Harvard ${ }^{6}$, Khan Academy ${ }^{7}$, Open Courseware at $\mathrm{MIT}^{8}$, $\mathrm{TED}^{5}$, and several others ${ }^{9,10}$. These videos allow students to learn and study at their own pace outside of the classroom or can be used to supplement lessons in the classroom. In addition, online videos are a useful reference material that students can review as needed later in their careers.

Videos have also been used to demonstrate a wide variety of experiments and techniques. For example, the Harvard BioVisions series includes videos on aseptic technique and DNA microarray experiments. ${ }^{6} \quad$ Several excellent experiment videos are also available on video sharing websites like Vimeo and YouTube (e.g. purification of green fluorescent protein by hydrophobic interaction chromatography ${ }^{11}$ ). The largest library of video experiments is provided by the Journal of Visualized Experiments (JOVE), a peer-reviewed online scientific video journal. Each JOVE video begins with an overview of relevant fundamental concepts, followed by a demonstration of the experiment and any relevant data analysis. In contrast to the Materials 
and Methods section of most journal articles, these videos show every step of the experiment in great detail to help scientists in other labs successfully conduct these experiments with high reproducibility. These videos can also be used in the classes to demonstrate applications of advanced concepts, thereby helping students to comprehend and appreciate the importance of such topics. Showing experiment videos is also much safer and costs much less than in-class demonstrations and especially appeals to students with a visual learning style.

The goal of this project was to develop three sets of videos: (1) reviews of fundamental biology concepts, (2) demonstrations of biological experiments, and (3) safety and basic skills training videos for students in lab classes. Each video was strategically designed to enhance student learning in lecture and/or lab-based courses by allowing students to review basic concepts and experimental protocols before class, thereby providing more time in class to discuss advanced concepts or perform experiments (see Figure 1). In addition, some of the experimental videos were also designed to enhance lecture-based classes by including experiment demonstrations that are typically too dangerous, time-consuming, or expensive to perform in a classroom. Two hypothetical applications of these videos are shown below.

1) In a lecture on recombinant proteins, an instructor may need to spend 10-20 minutes reviewing basic concepts like transcription and translation before introducing more advanced topics like fermentation. Our review videos for these basic concepts could be assigned before class to provide more time in class to discuss expression plasmids or demonstrate related techniques with short videos of actual experiments.

2) In lab courses, up to 30 minutes might be spent introducing or demonstrating how to conduct an experiment. Our experiment and training videos allow students to learn their protocols before class, thereby providing more time during the lab to actually conduct the experiments. In addition, students may also refer to the experiment videos during class to help them successfully complete the experiment or after lab while they are writing lab reports.

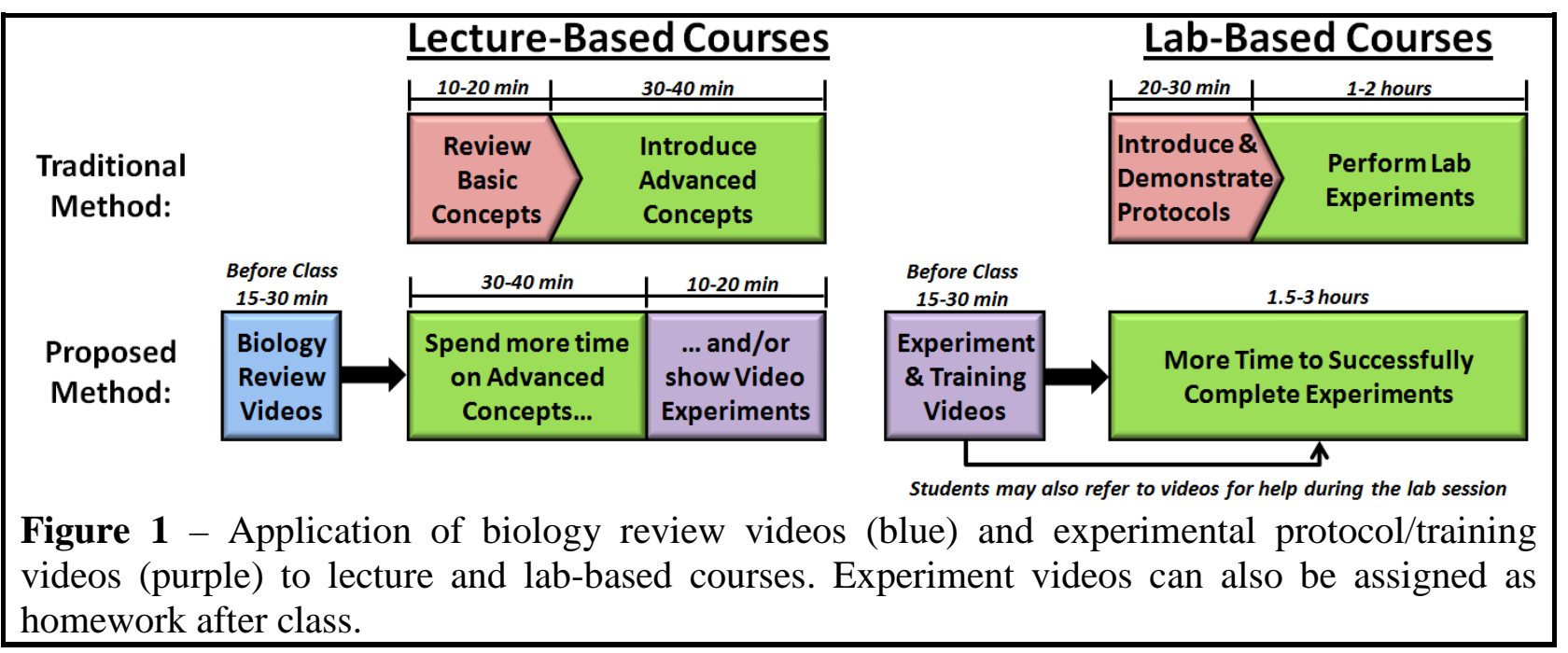




\section{Preparation of Videos}

Review Videos of Fundamental Biology Concepts: Each review video was recorded in a conventional distance learning classroom with a camera that simultaneously recorded the instructor, their PowerPoint slides, and any sketches or notes that were made in real time on the slides. All of the slides were archived and made available to students upon request. A comprehensive list of all the review videos and their content is shown in Table 1.

\begin{tabular}{|l|l|l|}
\hline Category & Video & Topics \\
\hline \multirow{4}{*}{$\begin{array}{l}\text { Molecular } \\
\text { Biology }\end{array}$} & Biomolecules & Structure and functions of lipids, sugars, proteins, and DNA \\
\cline { 2 - 3 } & Gene Expression & Transcription, translation, and regulation of gene expression \\
\cline { 2 - 3 } & DNA & DNA replication and structure \\
\cline { 2 - 3 } & Proteins & DNA, RNA, Amino acids, and levels of protein structure \\
\cline { 2 - 3 } $\begin{array}{l}\text { Cell Biology } \\
\text { /Physiology }\end{array}$ & Enzymes & Enzyme structure and reaction kinetics \\
\cline { 2 - 3 } & Cell Structure & Structures and functions of various organelles \\
\cline { 2 - 3 } & Metabolism & Glycolysis and the Kreb's Cycle \\
\cline { 2 - 3 } $\begin{array}{l}\text { Human } \\
\text { Anatomy }\end{array}$ & Cell Signaling & Receptors and signaling cascades (e.g. JAK/STAT) \\
\cline { 2 - 3 } & Immune System & Immune cells and activation pathways \\
\cline { 2 - 3 } & Cancer & Causes, effects, and treatments for cancer \\
\hline \multirow{2}{*}{ Microbiology } & Types of Cells & Bacteria, Fungi, Eukaryotes, and Viruses as well \\
\cline { 2 - 3 } & Plasmids & Elements required for recombinant protein expression \\
\hline
\end{tabular}

Table 1 - List of fundamental biology concepts covered in our review videos.

Experiment/Protocol Videos: Experimental procedures were recorded in a laboratory setting with a camcorder and tripod. Video clips were edited using Camtasia Studio (TechSmith) and integrated into PowerPoint presentation slides that included instructions for each step of the experiment, along with troubleshooting advice and other detailed notes. For example, one of the slides from the Restriction Digest experiment video is shown in Figure 2. While the video plays in the central panel, short step-by-step instructions are listed below it and more detailed notes are displayed in the panel on the right. In this case, the notes include a recipe for the digest reaction and important reminders about the buffers and times that should be used to optimize the reaction.

Video slides were also preceded by 1-2 introduction slides with computer animations that provided an overview of the experiment and any relevant concepts. The entire PowerPoint presentation was then narrated, converted to .mp4 video format, and uploaded to MediaSite. A list of the experiment and training videos that were produced is shown in Table 2.

\begin{tabular}{|l|l|l|}
\hline Category & Experiment/Topic & Description \\
\hline \multirow{4}{*}{$\begin{array}{l}\text { Training } \\
\text { Modules }\end{array}$} & Biohazard Safety & Common biohazards \& proper safety measures \\
\cline { 2 - 3 } & Basic Lab Skills & Pipetting, balances, centrifugation, etc. \\
\cline { 2 - 3 } & Aseptic Technique & Tips to avoid contamination of cell cultures \\
\cline { 2 - 3 } & Waste Disposal & Proper disposal of cells, liquids, and chemicals \\
\hline
\end{tabular}




\begin{tabular}{|c|c|c|}
\hline Animal & Freezing \& Thawing Cells & Freezing cells in DMSO and starting new lines \\
\hline Cell & Passaging Animal Cells & Trypsinization and counting cells \\
\hline Culture & $\mathrm{CO}_{2}$ Incubators and the BSC & Basic guidelines for culturing animal cells \\
\hline Bacterial & Bacterial Culture Techniques & Media prep, inoculation, and incubation of cells \\
\hline & Plasmid DNA Purification & Using a miniprep kit to isolate plasmid DNA \\
\hline Culture & Recombinant Protein Expression & Expression of the aeBlue protein in E. coli \\
\hline Micrn & Light Microscopes & Examining cells with an inverted microscope \\
\hline IVICroscopy & Fluorescent Microscopes & Imaging fluorescent animal cells \\
\hline & Overview of Cloning & Introduction to the molecular biology videos \\
\hline & PCR & Amplification of a target gene for cloning \\
\hline & Restriction Digests & Digestion of PCR products and plasmid DNA \\
\hline Iv & DNA Ligation & Joining DNA fragments to make a new plasmid \\
\hline & Bacterial Transformation & Inserting a DNA plasmid into E. coli cells \\
\hline & Agarose Gel Electrophoresis & Separation of DNA fragments on an agarose gel \\
\hline & Agarose Gel Extraction & Recovery of DNA fragments from a gel \\
\hline & Anion Exchange (AEX) & Purification of human hemoglobin \\
\hline & Hydrophobic Interaction (HIC) & Purification of recombinant aeBlue protein \\
\hline Durificat & Metal Affinity (IMAC) & Zinc affinity purification of human hemoglobin \\
\hline & Precipitation and Desalting & Selective precipitation of hemoglobin by $\mathrm{NiCl}_{2}$ \\
\hline Senaration & Ultrafiltration & Purification of erythrocruorin by $500 \mathrm{kDa}$ filters \\
\hline & PAGE & Separation of proteins by molecular weight \\
\hline & Dialysis & Assembly of a device to remove salt from water \\
\hline
\end{tabular}

Table 2 - List of experiments and training videos prepared in our project

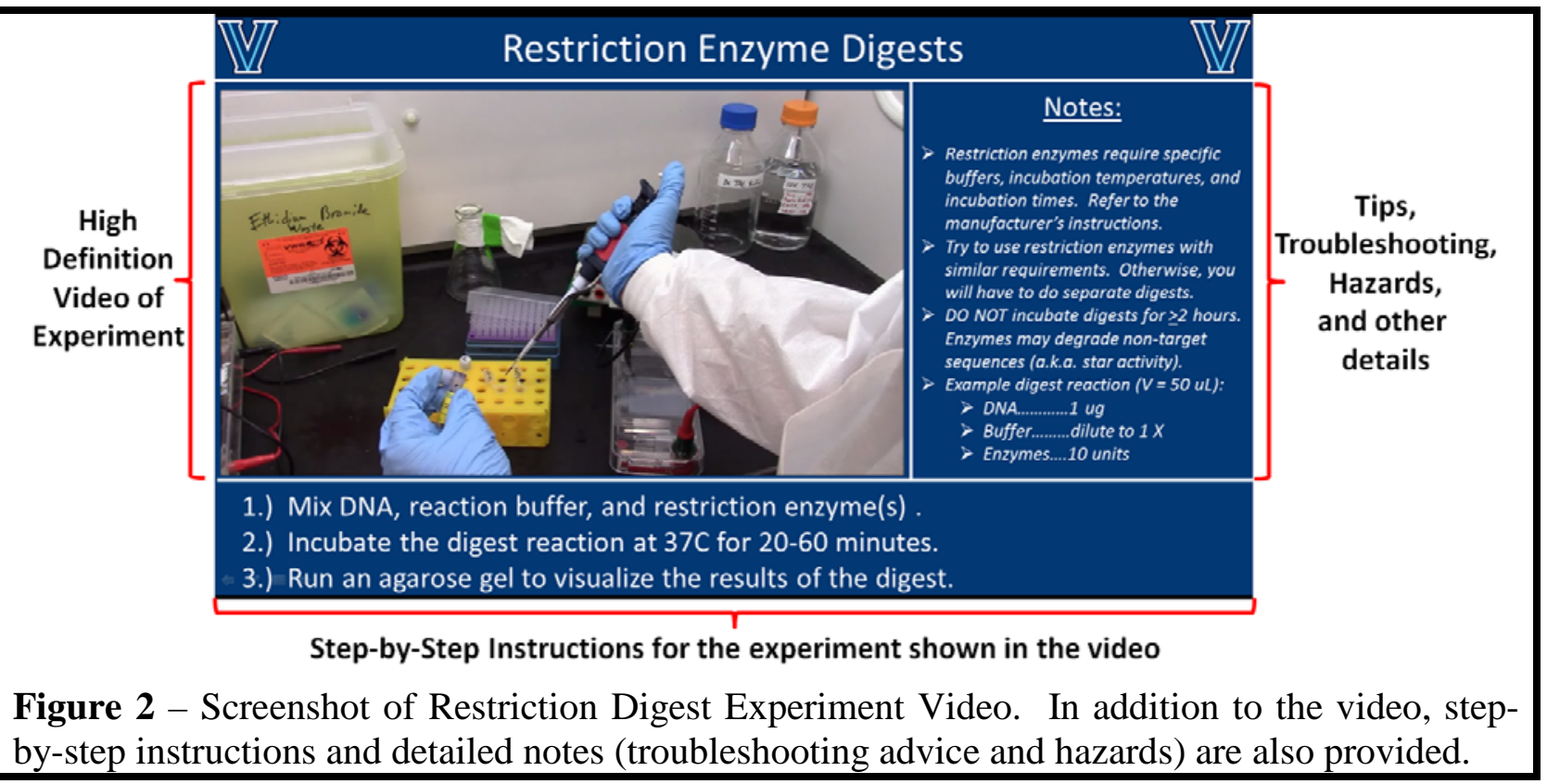

Note: All videos are available upon request - please contact the corresponding author (Dr. Jacob J. Elmer). 
Student Feedback Surveys: A survey with 7 questions and a space for suggestions or comments was sent to students after they watched each video. Comments and the responses to each question were compiled, analyzed, and saved in Excel. Examples of questions include:

- Was the video enjoyable? (No, Somewhat, and Very Much)

- Did the video help you understand material presented in class? (No, Somewhat, Yes)

- How would you describe the length of the video? (Too short, Too long, Appropriate)

- Will you use this video to study for the test? (No, Maybe, Yes)

- Would you recommend that this video be used again in this class? (No, Maybe, Yes)

\section{Applications of the Videos}

\section{Training Videos for Research Students}

At the beginning of the 2014-2015 school year, all undergraduate and graduate students participating in research in our department were required to watch the Biohazard Safety, Basic Lab Skills, and Waste Disposal training modules shown in Table 2. The students were then given three chances to take quizzes based on each video and were informed that they would not be allowed to begin their research until they had earned a perfect score on each quiz.

Most of the students passed each quiz on the first attempt (57-79\%), as shown in Figure 3. The biohazard safety quiz had the highest first attempt pass rate $(79 \%)$, while the waste disposal quiz had the highest amount of $2^{\text {nd }}$ and $3^{\text {rd }}$ attempts (29\% of students had to take the quiz a $2^{\text {nd }}$ time and $14 \%$ required a $3^{\text {rd }}$ attempt). No $4^{\text {th }}$ attempts were required on any quiz. There was a significant fraction of students that did have to make a second attempt on each quiz (21-29\%), but it is important to mention that most (13/14) of the quizzes that were not perfect on the first attempt only had a single mistake (9 out of 10 correct) and the most common mistake was to select only one answer in multiple choice questions with multiple correct answers (two questions of this type were on the Waste Disposal quiz). Overall, these results suggest that these training videos are an effective tool for teaching students basic lab skills and safety guidelines.

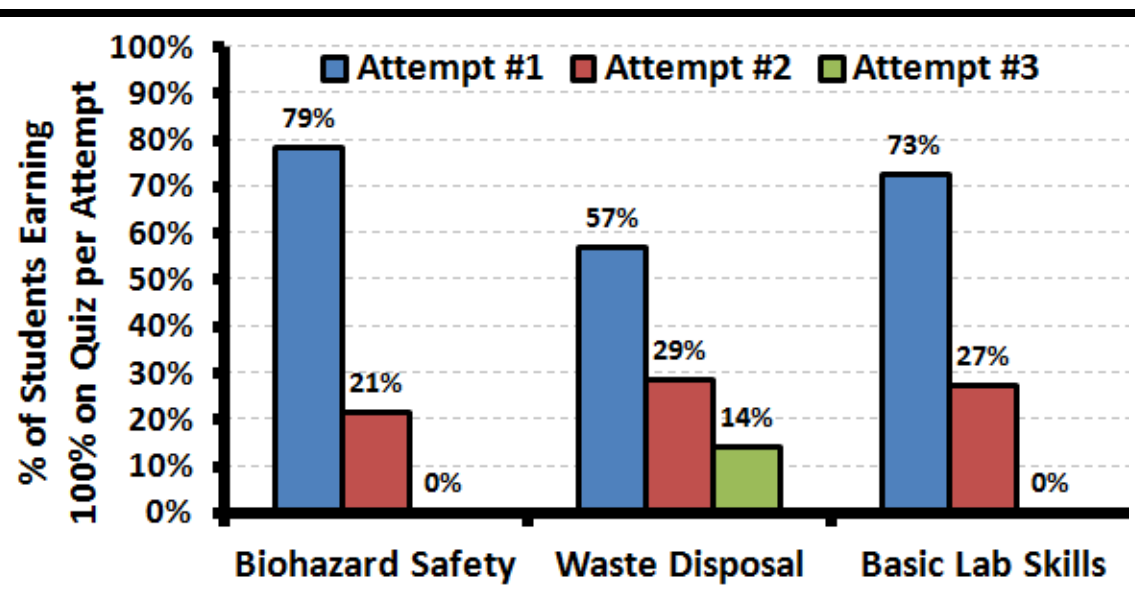

Figure 3 - 14 students were given 3 chances to pass quizzes based on 3 training videos. Blue bars show the percentage of students who passed the quiz on the first attempt, while red and green bars represent the percentage that passed on second and third attempts, respectively. 
While we found these training videos to be a convenient, consistent, and thorough way to train our students, it is important to mention that the videos are only a portion of our total training regimen. Once the students had passed their quizzes, we also gave them personal lab safety tours and demonstrated specific protocols to ensure that they were properly trained. Therefore, these training modules are not used to replace traditional training practices, but we have found them to be a good initial experience to start training our students before they begin working in the lab.

\section{Experiment Video in Biotechnology Classes:}

Two of the experiment videos - Overview of Cloning and Restriction Digests - were assigned as homework in an undergraduate elective course (Introduction to Biotechnology) and a required graduate course (Biochemical Engineering). These videos are specifically designed to show students how plasmids are prepared for recombinant protein expression and we assigned them as a supplement to in-class lessons that focused on bacterial fermentation and protein expression. This combination allowed students to see how plasmids are made in the videos and then discuss how to optimize protein expression from those plasmids during class.

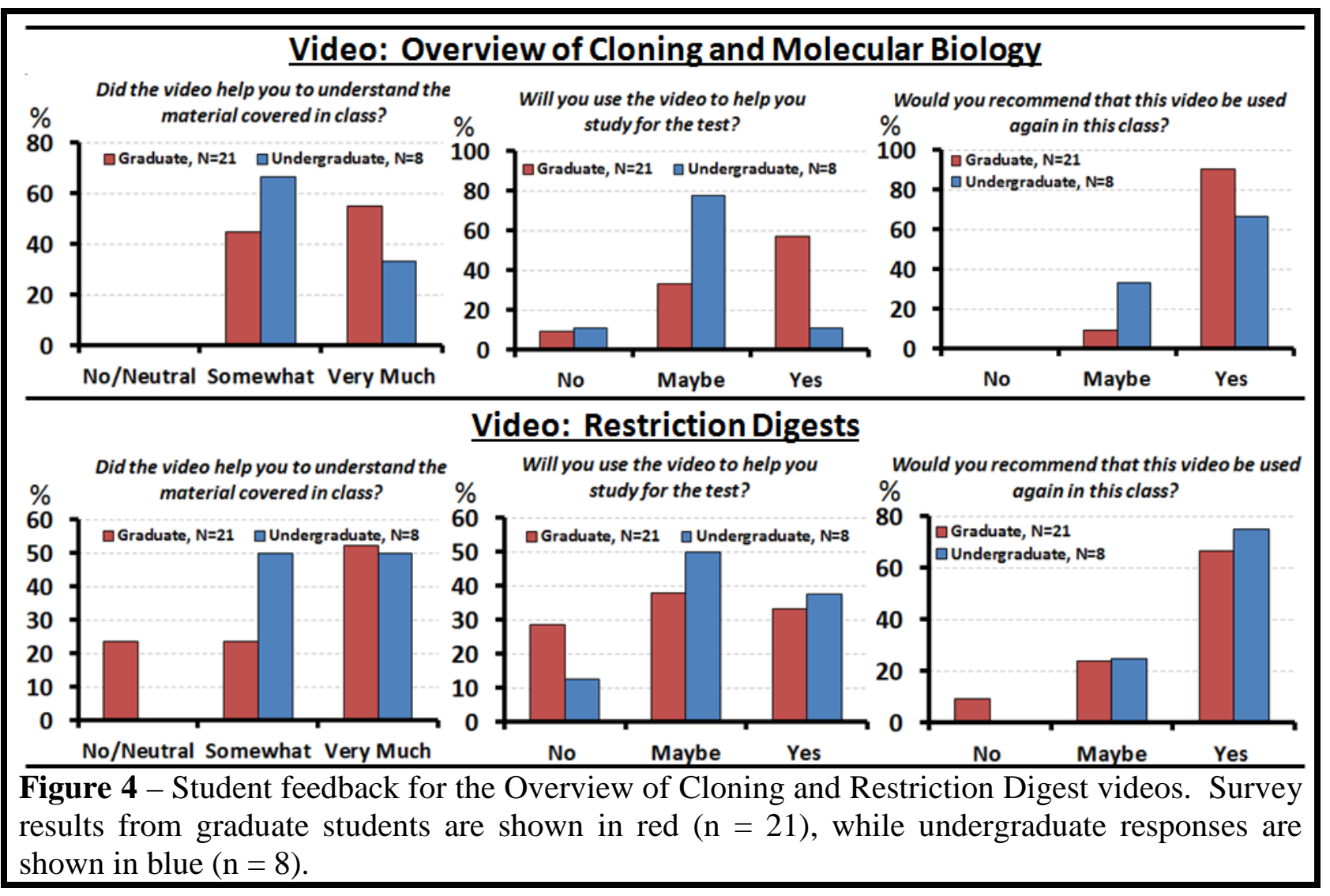

After the students had watched each video, they were asked to complete a brief survey with several questions and a space for comments and suggestions. The responses from graduate (red) and undergraduate (blue) students for three of the questions are shown in Figure 4. Students in both classes agreed that both of the videos helped them to better understand the 
material covered in class (somewhat or very much) and the majority of students also reported that they would or may use the videos to help them study for the next test. The students also recommended that the videos be offered again in the class next year. However, there was a significant fraction of graduate students who commented that the restriction digest video was too long (23.8\%, 5/21 students) and it did not help them to understand the material covered in class (23.8\%). Therefore, we may need to streamline and revise the content of the digest video for the graduate class, but the overall opinion of both videos seems to be highly positive in both classes.

\section{Protocol/Training Video in a Freshman Engineering Class}

As part of our freshman engineering program, students are required to take two halfsemester project-based courses in which they work on multi-disciplinary projects to design a product or system that addresses a real world problem. The goal of one of these projects is to construct a dialysis device using plastic tubing, a dialysis membrane, and $15 \mathrm{~mL}$ centrifuge tubes. $^{12}$ Although the design is fairly straightforward and the instructor demonstrates how to construct the device in class, the students' devices frequently have leaks or blockages that require them to start over and lose valuable time that could be used to test their devices. To address this problem, we prepared an instructional video that showed them how to construct the dialysis device and highlighted helpful tips and common pitfalls in the assembly process. Interestingly, many students started bringing their laptops to class to watch the video as they were assembling the device. Compared to previous semesters, the students that were given the instructional video built devices that had fewer flaws and were able to spend more time testing and collecting data (instead of rebuilding their devices).

\section{Video: Overview of Cloning and Molecular Biology}
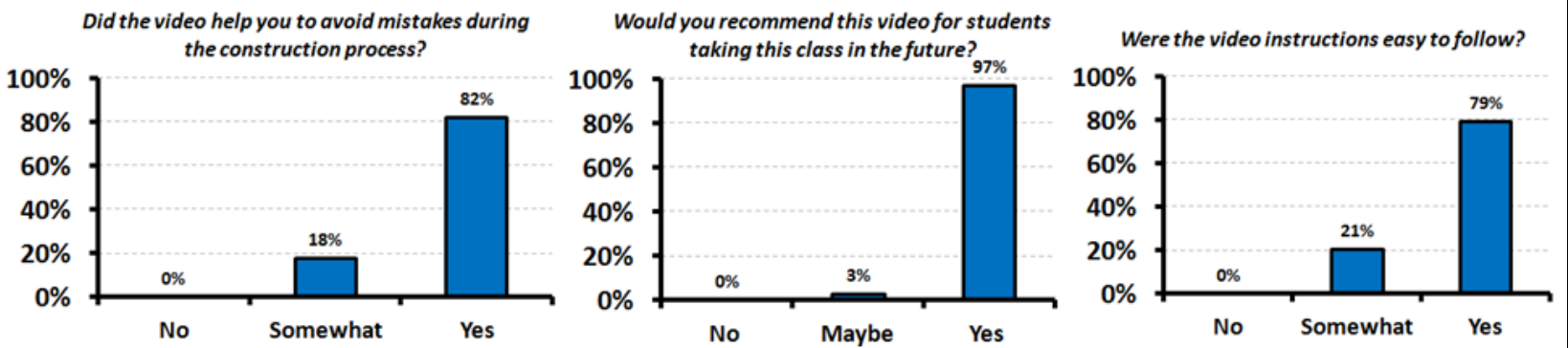

Figure 5 - Survey results for the Artificial Kidney Dialysis Module instructional video. Out of the 34 students in the class, a majority found the video helpful and recommend it for future use.

The results of student surveys for the instructional video were highly positive (see Figure 5). All of the students agreed that the video helped them to avoid mistakes during the construction process (82\% entirely, 18\% somewhat) and $97 \%$ of students recommended that this video be used again in future offerings of the class. However, there were several students (21\%, 7 out of 34) who indicated the video was only "somewhat" easy to follow. One key difference between this video and our other videos is that there was no narration or audio to accompany the video and step-by-step instructional captions. Interestingly, five of the seven students who chose "somewhat" as their response to this question also indicated in the comments section that video would be more effective with narration. These results show that while video alone is a powerful 
learning tool, audio or narration is also very important to some students (even though sufficient written instructions and captions are provided along with the video).

\section{Review Video on Proteins in a Protein Engineering Course}

The review video Introduction to Proteins was assigned to students before the first day of a graduate course on Protein Engineering. This review video is a brief (15 min) overview that discusses transcription, translation, amino acids, and protein structure (primary, secondary, tertiary, and quaternary). Assigning this video before class freed up extra time in our first lesson on advanced protein synthesis to discuss how tRNA's are charged with amino acids by amino acyl transferases and how this pathway can be modified to incorporate unnatural amino acids into proteins. While surveys (data not shown) indicated that only half of the class (6 out of 12 students) chose to watch the video, the students that did watch it all agreed that the review helped them to understand the content in class at least somewhat and that the video should be offered in again in future offerings of the course.

\section{Future Work}

Our current survey results indicate that our review and experiment videos are effective and well received by our students, but there is still much work to be done. Since a significant fraction of students have commented that they would prefer a shorter version of the experiment videos, we are currently preparing "abridged" versions of the experiment videos that are less than 5 minutes long and may be more appropriate for some classes that do not require the fine details of each experiment. Some of the shortened videos will also be converted into Graphics Interchange Format (GIF) files, allowing them to be easily incorporated into PowerPoint slides and shown in class as visual demonstrations. We may also disseminate these abridged videos and GIF's to high schools with advanced courses in biology or biotechnology to broaden the impact of these videos and increase interest in engineering and science.

Due to the success of these videos in our biochemical engineering courses, we are also planning experiment videos for other non-biological Chemical Engineering (ChE) courses like Unit Operations. These videos would show students how to operate several pieces of common equipment in our lab courses, including double-pipe heat exchangers, evaporators, and others.

\section{$\underline{\text { Conclusion }}$}

Over the course of this project, we developed 14 review videos for fundamental biology concepts and 25 experiment videos showing a wide range of biological experiments and techniques. We have already implemented 7 of these videos in classes and labs over the past year and observed the following benefits:

- Assigning review videos before class provides more time for advanced concepts in class

- Students appreciate seeing real world applications of concepts in the experiment videos

- Experiment videos also help students to understand the related concepts covered in class

- Training and instructional videos help students to successfully complete and prepare for lab experiments 
Since our survey data indicates that these videos are effective learning tools, we will continue assigning the remaining videos in Tables 1 and 2 throughout the rest of our curriculum. We will also continue collecting survey data to help us revise and optimize each video with the ultimate goal of creating a BioChE review/experiment video library that can be disseminated to other colleges and high schools. These videos will be made available upon request to the corresponding author (Dr. Jacob J. Elmer).

\section{Bibliography}

1. Herreld, C. F. \& Schiller, N. A. Case Studies and the Flipped Classroom. J. Coll. Sci. Teach. 42, 62-66 (2013).

2. Williams, S. L. The Effectiveness of Distance Education in Allied Health Science Programs: A MetaAnalysis of Outcomes. Am. J. Distance Educ. 20, 127-141 (2006).

3. $\quad$ Stith, B. J. Use of animation in teaching cell biology. Cell Biol. Educ. 3, 181-8 (2004).

4. Sanger, M. J., Brecheisen, D. M. \& Hynek, B. M. Can Computer Animations Affect College Biology Students' Conceptions about Diffusion and Osmosis? Am. Biol. Teach. 63, 104-109 (2001).

5. Animations of Unseeable Biology. at $<0$ http://www.ted.com/talks/drew_berry_animations_of_unseeable_biology.html>

6. BioVisions at Harvard University. at <multimedia.mcb.harvard.edu>

7. The Khan Academy. at <www.khanacademy.org >

8. MIT Open CourseWare - Courses in the Biology Department. at <http://ocw.mit.edu/courses/\#biology $>$

9. $\quad$ Kyrk, J. Cell Biology Animation. at <www.johnkyrk.com>

10. Free Biology Animations. at <http://www.learnerstv.com/animation/Free-biology-animations-page1.htm>

11. BioNetwork. Hydrophobic Interaction Chromatography (GFP Purification Part 4 of 6). (2014).

12. Comolli, N., Kelly, W. \& Wu, Q. The Artificial Kidney: Investigating Current Dialysis Methods As A Freshman Design Project. in Am. Soc. Eng. Educ. (2010). 